\title{
Strong Prime LI-ideals in Lattice Implication Algebras
}

\author{
Lijun Bai Yang Xu Zhiyan Chang Weitao Xu \\ Department of Mathematics, Southwest Jiaotong University, Chengdu 610031, P.R. China
}

\begin{abstract}
In this paper, the notion of strong prime $L I$-ideals (briefly, SPLI-ideals) of lattice implication algebras is introduced. The relations between SPLI-ideals and prime $L I$-ideals, between SPLI-ideals and maximal proper $L I$-ideals, between SPLI-ideals and the finite union property, and between ultra-filter and $S P L I$-ideal are investigated. Finally, we conclude that $S P L I$-ideals are equivalent to maximal proper $L I$-ideals.
\end{abstract}

Key words: Lattice implication algebra, Prime $L I$-ideal, SPLI-ideal, Finite union property

\section{Introduction}

Non-classical logic has become a considerable formal tool for computer science and artificial intelligence to deal with fuzzy information and uncertainty information. Many-valued logic, a great extension and development of classical logic, has always been a crucial direction in non-classical logic. In order to research the many-valued logical system whose propositional value is given in a lattice, in $1990 \mathrm{Xu}$ [1] proposed the concept of lattice implication algebra. Since then this logical algebra has been extensively investigated by several researchers(see e.g. [12]-[17]). In [9] Xu and Qin introduced the notions of filters and implicative filters in lattice implication algebras, and investigated their some properties. In a lattice implication algebra, filters are important substructures, they play a significant role in studying the structure and the properties of lattice implication algebras. In[11], Jun et al. introduced the notions of positive implicative filters and associative filters in lattice implication algebras, and investigated their some properties. In[4], Jun et al. defined the notion of $L I$-ideals in lattice implication algebras and investigated its some properties. In[5], Jun defined the notion of prime $L I$-ideals in lattice implication algebras and investigated its some properties. In this paper, as an extension of above-mention work we introduce the notions of strong prime $L I$-ideals in lattice implication algebras, and investigated its some properties. In Secation 2, we list some basic information on the lattice implication algebras which is needed for development of this topic. In Section 3, we introduce the notion of the union property of lattice implication algebras. We give the sufficient and necessary condition that a proper $L I$-ideal to have the union property. In section 4 , we introduce the notion of strong prime $L I$-ideals (briefly, SPLI-ideals) of lattice implication algebras, and talk about the relations between $S P L I$-ideals and prime $L I$-ideals, between $S P L I$-ideals and maximal proper $L I$-ideals, between $S P L I$-ideals and the finite union property, and between ultra-filter and SPLI-ideal. We prove that $S P L I$-ideals are equivalent to maximal proper $L I$-ideals.

\section{Preliminaries}

Definition 2.1[1] Let $(L, \vee, \wedge, O, I)$ be a bounded lattice with an order-reversing involution ', $I$ and $O$ the greatest and the smallest element of $L$ respectively, and

$$
\rightarrow: L \times L \rightarrow L
$$

be a mapping. ( $\left.L, \vee, \wedge,{ }^{\prime}, \rightarrow, O, I\right)$ is called a lattice implication algebra if the following conditions hold for any $x, y, z \in L$ :

$$
\begin{aligned}
& \left(\mathrm{L}_{1}\right) x \rightarrow(y \rightarrow z)=y \rightarrow(x \rightarrow z), \\
& \left(\mathrm{L}_{2}\right) x \rightarrow x=I, \\
& \left(\mathrm{~L}_{3}\right) x \rightarrow y=y^{\prime} \rightarrow x^{\prime}, \\
& \left(\mathrm{L}_{4}\right) \text { If } x \rightarrow y=y \rightarrow x=I, \text { then } x=y, \\
& \left(\mathrm{~L}_{5}\right)(x \rightarrow y) \rightarrow y=(y \rightarrow x) \rightarrow x, \\
& \left(\mathrm{~L}_{6}\right)(x \vee y) \rightarrow z=(x \rightarrow z) \wedge(y \rightarrow z), \\
& \left(\mathrm{L}_{7}(x \wedge y) \rightarrow z=(x \rightarrow z) \vee(y \rightarrow z),\right.
\end{aligned}
$$

A lattice implication algebra is called a lattice $H$ implication algebra If it satisfies

$$
x \vee y \vee((x \wedge y) \rightarrow z)=I .
$$

In a lattice implication algebra $L$, [3] defines two binary operations $\otimes$ and $\oplus$ as follows: for any $x, y \in L$,

$$
\begin{gathered}
x \otimes y=\left(x \rightarrow y^{\prime}\right)^{\prime}, \\
x \oplus y=x^{\prime} \rightarrow y .
\end{gathered}
$$

In a lattice implication algebra $L$, the following hold:

$\left(\mathrm{L}_{8}\right) x \rightarrow(y \rightarrow z)=(x \otimes y) \rightarrow z$,

$\left(\mathrm{L}_{9}\right)(x \oplus y)^{\prime}=x^{\prime} \otimes y^{\prime}$,

$\left(\mathrm{L}_{10}\right)(x \otimes y)^{\prime}=x^{\prime} \oplus y^{\prime}$,

$\left(\mathrm{L}_{11}\right) O \otimes x=O, I \otimes x=x, x \otimes x^{\prime}=O$, 
$\left(\mathrm{L}_{12}\right) O \oplus x=x, I \oplus x=I, \quad x \oplus x^{\prime}=I$,

$\left(\mathrm{L}_{13}\right) O \rightarrow x=I, x \rightarrow O=x^{\prime}$,

$\left(\mathrm{L}_{14}\right) I \rightarrow x=x, \quad x \rightarrow I=I$,

$\left(\mathrm{L}_{15}\right) x \leq y$ if and only if $x \rightarrow y=I$.

Definition 2.2 [3] Let $L$ be a lattice implication algebra. An $L I$-ideal $A$ is a non-empty subset of $L$ such that for any $x, y \in L$,

$\left(\mathrm{I}_{1}\right) O \in A$,

$\left(\mathrm{I}_{2}\right)(x \rightarrow y) \in A$ and $y \in A$ imply $x \in A$.

In a lattice implication algebra, $A \subseteq L$, the least $L I$-ideal containing $A$ is called the $L I$-ideal generated by $A$ and denoted by $\langle A\rangle$. Specially, if $A=\{a\}$, we write $\langle\{a\}\rangle$ as $\langle a\rangle$.

Definition 2.3 [9] Let $L$ be a lattice implication algebra, $J \subseteq L$ is said to be a filter of $L$, if it satisfies the following conditions:

$\left(\mathrm{J}_{1}\right) I \in J$,

$\left(\mathrm{J}_{2}\right)$ for any $x, y \in L \quad$ if $\quad x \in J$ and $(x \rightarrow y) \in J$, then $y \in J$.

For any non-empty subset $A$ of a lattice implication algebra, let

$$
A^{\prime}=\left\{x^{\prime} \mid x \in A\right\} \text {. }
$$

We show the relation between $L I$-ideal and filter of lattice implication algebra.

Theorem 2.4 [3] Let $A$ be a non-empty subset of a lattice implication algebra $L$. then $A$ is a filter of $L$ if and only if $A^{\prime}$ is an $L I$-ideal of $L$.

Definition 2.5 [10] Let $L$ be a lattice implication algebra, a filter $J$ of $L$ is called an ultra-filter if for any $x \in L, x \in J$ if and only if $x^{\prime} \notin J$.

Definition 2.6 [5] Let $L$ be a lattice implication algebra, $P$ a proper $L I$-ideal of $L$. $P$ is called a prime $L I$-ideal if $x \wedge y \in P$ implies $x \in P$ or $y \in P$.

Definition 2.7 [3] Let $L_{1}$ and $L_{2}$ be lattice implication algebras, $f: L_{1} \rightarrow L_{2}$ a mapping from $L_{1}$ to $L_{2}$, if

$$
f(x \rightarrow y)=f(x) \rightarrow f(y)
$$

holds for any $x, y \in L_{1}$, then $f$ is called an implication homomorphism from $L_{1}$ to $L_{2}$. If an implication homomorphism $f$ is a surjection, then it is called an implication epimorphism. If $f$ is an implication homomorphism and satisfies

$$
\begin{gathered}
f(x \vee y)=f(x) \vee f(y), \\
f(x \wedge y)=f(x) \wedge f(y), \\
f\left(x^{\prime}\right)=(f(x))^{\prime},
\end{gathered}
$$

then $f$ is called a lattice implication homomorphism from $L_{1}$ to $L_{2}$.

\section{The union property of lattice implication algebras}

Definition 3.1 Let $L$ be a lattice implication algebra. $A \subseteq L$ is said to have the union property if for any $a_{1}, a_{2}, \ldots, a_{n} \in A, \quad a_{1} \oplus a_{2} \oplus \cdots \oplus a_{n}<I$.

In what follows,

$\left[a_{1}, a_{2}, \cdots, a_{n}, x\right]^{\Delta}=a_{1} \rightarrow\left(a_{2} \rightarrow\left(\cdots \rightarrow\left(a_{n} \rightarrow x\right) \cdots\right)\right)$.
Specially,

$[a, x]^{0} \stackrel{\Delta}{=} x$,

$[a, x]^{1} \stackrel{\Delta}{=}[a, x]=a \rightarrow x$,

$[a, x]^{n \stackrel{\Delta}{=}} a \rightarrow(a \rightarrow(\cdots \rightarrow(a \rightarrow x) \cdots)) \quad n \geq 2$.

Lemma 3.2 [3] If $A$ is a non-empty subset of a lattice implication algebra $L$, then

$\langle A\rangle=\left\{x \in L \mid\left[a_{1}^{\prime}, \cdots, a_{n}^{\prime}, x^{\prime}\right]=I\right.$, for some
$\left.a_{1}, \cdots, a_{n} \in A\right\}$.

Theorem 3.3 let $L$ be a lattice implication algebra, $\varnothing \neq A \subset L$. Then

$\langle A\rangle=\left\{x \mid x \in L\right.$, there exist $a_{1}, \cdots, a_{n} \in A$, s.t., $\left.a_{1} \oplus \cdots \oplus a_{n} \geq x\right\}$.

Proof. By

$$
\begin{aligned}
& {\left[a_{1}^{\prime}, \cdots, a_{n}^{\prime}, x^{\prime}\right]=I } \\
\Leftrightarrow & a_{1}^{\prime} \rightarrow\left(\cdots \rightarrow\left(a_{n}^{\prime} \rightarrow x^{\prime}\right) \cdots\right)=I \\
\Leftrightarrow & \left(a_{1}^{\prime} \otimes \cdots \otimes a_{n}^{\prime}\right) \rightarrow x^{\prime}=I \quad\left(\text { by } \mathrm{L}_{8}\right) \\
\Leftrightarrow & \left(a_{1} \oplus \cdots \oplus a_{n}\right)^{\prime} \rightarrow x^{\prime}=I \quad\left(\text { by } \mathrm{L}_{9}\right) \\
\Leftrightarrow & x \rightarrow\left(a_{1} \oplus \cdots \oplus a_{n}\right)=I \\
\Leftrightarrow & a_{1} \oplus \cdots \oplus a_{n} \geq x,
\end{aligned}
$$

and by Lemma 3.2, we complete the proof.

Theorem 3.4 Let $L$ be a lattice implication algebra. $A \subseteq L$, then $\langle A\rangle$ is a proper $L I$-ideal if and only if $A$ has the finite union property.

Proof. Suppose $\langle A\rangle$ is a proper $L I$-ideal, and it doesn't have the finite union property. So there exist $a_{1}, a_{2}, \ldots, a_{n} \in A$, s.t., $a_{1} \oplus a_{2} \oplus \cdots \oplus a_{n} \geq I$, by Theorem 3.3, $I \in\langle A\rangle$, contradiction.

Conversely, suppose that $A$ has the finite union property, then for any $a_{1}, a_{2}, \ldots, a_{n} \in A$, $a_{1} \oplus a_{2} \oplus \cdots \oplus a_{n}<I$, so $I \notin A$ and $A$ is a proper LI-ideal.

Theorem 3.5 Let $L$ be a lattice implication algebra, $a, b, x \in L$.

(1) If $a \geq b$, then $\left[a^{\prime}, x^{\prime}\right]^{n} \geq\left[b^{\prime}, x^{\prime}\right]^{n}$ for any $n \in N$,

(2) If $n, m \in N, n \geq m$, then $\left[a^{\prime}, x^{\prime}\right]^{n} \geq\left[a^{\prime}, x^{\prime}\right]^{m}$,

(3) $\left[a^{\prime}, x^{\prime}\right]^{n} \geq x^{\prime}$ for any $n \in N$.

Proof. These conclusions are trivial when $n=0$ or $\mathrm{m}=0$.

(1) we use induction over $\mathrm{n}$ to show 
$\left[a^{\prime}, x^{\prime}\right]^{n} \geq\left[b^{\prime}, x^{\prime}\right]^{n}$. If $\mathrm{n}=1$, then

$\left[a^{\prime} \rightarrow x^{\prime}\right]=a^{\prime} \rightarrow x^{\prime}=x \rightarrow a \geq x \rightarrow b=b^{\prime} \rightarrow x^{\prime}=\left[b^{\prime}, x^{\prime}\right]$

Suppose now $\mathrm{n}>1$, and $\left[a^{\prime}, x^{\prime}\right]^{m} \geq\left[b^{\prime}, x^{\prime}\right]^{m}$ for any $\mathrm{m}<\mathrm{n}$, then when $\mathrm{n}=\mathrm{m}+1$,

$\left[a^{\prime}, x^{\prime}\right]^{m+1}=a^{\prime} \rightarrow\left[a^{\prime}, x^{\prime}\right]^{m} \geq a^{\prime} \rightarrow\left[b^{\prime}, x^{\prime}\right]^{m} \geq b^{\prime} \rightarrow\left[b^{\prime}, x^{\prime}\right]^{m}=\left[b^{\prime}, x^{\prime}\right]^{m+1}$

(2) Suppose that $\mathrm{n}=\mathrm{m}+\mathrm{p}$, it follows that $p \geq 0$. We use induction over $\mathrm{p}$ to show $\left[a^{\prime}, x^{\prime}\right]^{m+p} \geq\left[a^{\prime}, x^{\prime}\right]^{m}$. If $\mathrm{p}=0$, then $\left[a^{\prime}, x^{\prime}\right]^{m+p} \geq\left[a^{\prime}, x^{\prime}\right]^{n+}$ holds.

Suppose now $\mathrm{p}=1$, then

$\left[a^{\prime}, x^{\prime}\right]^{m+p}=a^{\prime} \rightarrow\left[a^{\prime}, x^{\prime}\right]^{m} \geq O^{\prime} \rightarrow\left[a^{\prime}, x^{\prime}\right]^{m}=\left[a^{\prime}, x^{\prime}\right]^{m}$.

Suppose now $\mathrm{p}>1$, and $\left[a^{\prime}, x^{\prime}\right]^{m+q} \geq\left[a^{\prime}, x^{\prime}\right]^{m}$ for any $\mathrm{q}<\mathrm{p}$. It follows that

$\left[a^{\prime}, x^{\prime}\right]^{m+p}=a^{\prime} \rightarrow\left[a^{\prime}, x^{\prime}\right]^{m+(p-1)} \geq a^{\prime} \rightarrow\left[a^{\prime}, x^{\prime}\right]^{m} \geq\left[a^{\prime}, x^{\prime}\right]^{m}$

(3) When $\mathrm{n}=1$, then

$\left[a^{\prime}, x^{\prime}\right]=a^{\prime} \rightarrow x^{\prime}=x \rightarrow a \geq x \rightarrow O=x^{\prime}$.

Suppose that $\mathrm{n}=\mathrm{m}$, and $\left[a^{\prime}, x^{\prime}\right]^{m} \geq x^{\prime}$ holds for any $m \in N$. It follows that

$\left[a^{\prime}, x^{\prime}\right]^{m+1}=a^{\prime} \rightarrow\left[a^{\prime}, x^{\prime}\right]^{m} \geq O^{\prime} \rightarrow\left[a^{\prime}, x^{\prime}\right]^{m} \geq O^{\prime} \rightarrow x^{\prime}=x^{\prime}$ Complete the proof.

\section{SPLI-ideals of lattice implication algebras}

Definition 4.1 Let $L$ be a lattice implication algebra. A proper $L I$-ideal $A$ is said to be a strong prime $L I$-ideal (briefly, SPLI-ideal) if $\left(x \rightarrow y^{\prime}\right)^{\prime} \in A$

$(x \otimes y \in A)$ implies $x \in A$, or $y \in A$ for any $x, y \in L$.

The relation between SPLI-ideals and prime $L I$-ideals in lattice implication algebras is as follows:

Theorem 4.2 A SPLI-ideal is a prime $L I$-ideal.

Proof. Let $A$ be a $S P L I$-ideal, we need to prove that if $x \wedge y \in A$ implies $x \in A$ or $y \in A$. In fact, by $x \otimes y \leq x \wedge y \leq x \vee y \leq x \oplus y$, we get $x \otimes y \in A$, because $A$ is a $S P L I$-ideal, so $x \in A$ or $y \in A$.

The relation between $S P L I$-ideals and maximal proper $L I$-ideals in lattice implication algebras is as follows:

Theorem 4.3 Let $L$ be a lattice implication algebra, $A \subseteq L$. The following statements are equivalent:

$\left(\mathrm{I}_{1}\right)$ A is a SPLI-ideal;

$\left(\mathrm{I}_{2}\right) A$ is a maximal proper $L I$-ideal.

Proof. $\left(\mathrm{I}_{1}\right) \Rightarrow\left(\mathrm{I}_{2}\right)$. Suppose that $A$ is a $S P L I$-ideal, so $A$ is a proper $L I$-ideal, if $A \subset B$ and $B$ is also a proper $L I$-ideal, we need to prove $A=B$. In fact, if there exist $x \in B$ such that $x \notin A$, then by $x \otimes x^{\prime}=O \in A$, so $x^{\prime} \in A \subset B$, i.e $(I \rightarrow x) \in B$, it follows that $I \in B$, and $B=L$, which is a contradiction.

$\left(\mathrm{I}_{2}\right) \Rightarrow\left(\mathrm{I}_{1}\right)$. Suppose $A$ is a maximal proper $L I$-ideal. We need to prove that $x \otimes y \in A$ implies $x \in A$, or $y \in A$ for any $x, y \in L$. Otherwise, if $x \otimes y \in A$, but $x \notin A$ and $y \notin A$. Let $B=A \bigcup\{x\}, D=\langle B\rangle$, we shall prove that $B$ has the union property. In fact, for any $y_{1}, \ldots y_{n} \in B$,

(a) If $y_{1}, \ldots, y_{n} \in A$, then $y_{1} \oplus \ldots \oplus y_{n} \in A$ by $L I$-ideals are closed with the operation $\oplus$, it follows that $y_{1} \oplus \ldots \oplus y_{n}<I$ because $A$ is a proper $L I$-ideal.

(b) If there exist $i<n$ such that $y_{i}=x$, without losing generality suppose $y_{1}=x$. If

$$
\begin{aligned}
y_{1} \oplus \ldots \oplus y_{n} & =x \oplus y_{2} \oplus \ldots \oplus y_{n} \\
& =x \rightarrow\left(y_{2} \oplus \ldots \oplus y_{n}\right) \\
& =I
\end{aligned}
$$

then $x^{\prime} \leq y_{2} \oplus \ldots \oplus y_{n}$, so $x^{\prime} \in A$. By supposition, $x \otimes x^{\prime}=O \in A$ implies $x \notin A$ and $x^{\prime} \notin A, \quad$ a contradiction.

By (a) and (b), we have proved that $B$ has the finite union property, so $\langle B\rangle$ is a proper $L I$-ideal, it follows by $A \subset\langle B\rangle$ that $A=\langle B\rangle$, i.e $A=\langle A \cup\{x\}\rangle$, that is $x \in A$, conflict.

The relation between $S P L I$-ideals and the finite union property in lattice implication algebras is as follows:

Theorem 4.4 Let $L$ be a lattice implication algebra, $A \subseteq L$. If $A$ has the finite union property, then there exist a $S P L I$-ideal $B$ such that $A \subseteq B$.

Proof. Let

$$
E=\{B \mid A \subset B, B \text { is a prime } L I \text {-ideal of } L\} .
$$

It follows that $E \neq \varnothing$ because $\langle A\rangle \in E$. Suppose that $B_{i} \in E$ for any $i<k$ such that:

$B_{1} \subseteq B_{2} \subseteq \cdots \subseteq B_{i} \subseteq \cdots$ let $B=\bigcup_{i<k} B_{i}$, it follows

that:

(1) $A \subset B$;

(2) $I \notin B$ because $I \notin B_{i}$ for any $i<k$;

(3) $O \in B$;

(4) if $y,(x \rightarrow y)^{\prime} \in B$, then there exists $i<k$ such that $y,(x \rightarrow y) \in B_{i}$, it follows that $x \in B_{i} \subseteq B$. So, $B$ is a proper $L I$-ideal and $B \in E$. It follows by Zorn's Lemma that $E$ has a maximal element $B$. Thus $B$ is a strong prime $L I$-ideal such that $A \subseteq B$.

From the theorem 4.4, we have the following corollary.

Corollary 4.5 Any proper $L I$-ideal of $L$ can be extended to a $S P L I$-ideal.

Theorem 4.6 If $A$ is a prime $L I$-ideal of lattice $H$ implication algebra $L$, then $A$ is a $S P L I$-ideal.

Proof. Suppose that $A$ is not a SPLI-ideal of $L$. Then there exist a proper $L I$-ideal $F$ of $L$ such that $A \subset F$ and $A \neq F$. It follows that there exists an element $a \in F$ such that $a \notin A$. We get $a \wedge a^{\prime}=O \in A$, it follows that $a^{\prime} \in A \subset F$, this implies that $a \vee a^{\prime}=I \in F$, which contradicts to that $F$ is a proper $L I$-ideal.

From the theorem 4.6, we have the following corollary.

Corollary 4.7 In a lattice $H$ implication algebra, the concept of prime $L I$-ideal and SPLI-ideal coincide.

Theorem 4.8 Let $L_{1}$ and $L_{2}$ be lattice implication algebras, $f: L_{1} \rightarrow L_{2}$ is a lattice implication 
homomorphism from $L_{1}$ to $L_{2}$. If $A$ is a SPLI-ideal of $L_{2}$, then $f^{-1}(A)$ is a SPLI-ideal of $L_{1}$.

Proof. For any $x, y \in L_{1}$, if

$$
\left(x \rightarrow y^{\prime}\right)^{\prime} \in f^{-1}(A)
$$

then

$$
f\left(\left(x \rightarrow y^{\prime}\right)^{\prime}\right) \in A,
$$

by Definition 2.7, we have

$$
\left(f\left(x \rightarrow y^{\prime}\right)\right)^{\prime} \in A,
$$

i.e.

$$
\left(f(x) \rightarrow f\left(y^{\prime}\right)\right)^{\prime} \in A
$$

and then

$$
\left(f(x) \rightarrow(f(y))^{\prime}\right)^{\prime} \in A
$$

for $A$ is a $S P L I$-ideal of $L_{2}$, then $f(x) \in A$ or $f(y) \in A$, so $x \in f^{-1}(A)$ or $y \in f^{-1}(A)$. By Definition 4.1, $f^{-1}(A)$ is a SPLI-ideal of $L_{1}$.

Lemma 4.9 [3] Let $L$ be a lattice implication algebra, $J$ a proper filter of $L . J$ is an ultra-filter if and only if $x \oplus y \in J$ implies $x \in J$ or $y \in J$ for any $x, y \in L$.

Finally, we give the relation between ultra-filter and SPLI-ideal as follows:

Theorem 4.10 Let $L$ be a lattice implication algebra, $A$ is a non-empty subset of $L$, let

$$
A^{\prime}=\left\{x^{\prime} \mid x \in A\right\}
$$

then $A$ is an ultra-filter if and only if $A^{\prime}$ is a $S P L I$-ideal of $L$.

Proof. Suppose that $A$ is an ultra-filter. For any $x^{\prime}, y^{\prime} \in L$, if $x^{\prime} \otimes y^{\prime} \in A^{\prime}$, i.e., $(x \oplus y)^{\prime} \in A^{\prime}\left(\right.$ by $\left.\mathrm{L}_{9}\right)$, then $x \oplus y \in A$. By Lemma 4.9, we get $x \in A$ or $y \in A$, and hence $x^{\prime} \in A^{\prime}$ or $y^{\prime} \in A^{\prime}$. So by Definition 4.1, $A^{\prime}$ is a SPLI-ideal.

Conversely, suppose that $A^{\prime}$ is a SPLI-ideal, for any $x, y \in L$, if $x \oplus y \in A$, then $(x \oplus y)^{\prime} \in A^{\prime}$, i.e., $x^{\prime} \oplus y^{\prime} \in A^{\prime}$, so $x^{\prime} \in A^{\prime}$ or $y^{\prime} \in A^{\prime}$. Hence $x \in A$ or $y \in A$, and $A$ is an ultra-filter.

\section{Conclusions}

In order to research the many-valued logical system whose propositional value is given in a lattice, $\mathrm{Xu}$ initiated the concept of lattice implication algebra. Hence for development of this many-valued logical system, it is needed to make clear the structure of an algebraic system. It is well known that to investigate the structure of an algebraic system, the ideals with special properties play an important role. In this paper, we proposed the notion of strong prime $L I$-ideals (SPLI-ideals) in lattice implication algebras, discussed the relations between SPLI-ideals and prime $L I$-ideals, between $S P L I$-ideals and maximal proper $L I$-ideals, and between ultra-filter and $S P L I$-ideal. We finally concluded that SPLI-ideals are equivalent to maximal proper $L I$-ideals. Actually, SPLI-ideal is the dual of ultra-filter. It hope above work would serve as a foundation for further study the structure of lattice implication algebras and develop corresponding many-valued logical system.

\section{Acknowledgement}

The work was partially supported by the National Natural Science Foundation of P. R. China (Grant No. 60474022) and the Research Fund for the Doctoral Program of Higher Education (Grant No. 20060613007).

\section{References}

[1] Y. Xu, Lattice implication algebra, Journal of Southwest Jiaotong university, 28: 20-27 (in Chinese), 1993.

[2] J. Liu, Studying on the structure of lattice implication algebra, Southwest Jiaotong Univ, 1996 (in Chinese).

[3] Y. Xu, D. Ruan, K.Y Qin and J. Liu, Lattice-Valued Logic, Springer-Verlag, 2003.

[4] Y. B. Jun, E. H. Roh and Y. Xu, LI-ideals in lattice implication algebras, Bull Korean Math Soc, 35: 13-24, 1998.

[5] Y. B. Jun, On $L I$-ideals and prime $L I$-ideals of lattice implications algebras, Korean Math. Soc. 36: 369-380, 1999.

[6] Y. B. Jun and Y. Xu, Fuzzy LI-ideals in lattice implication algebras, Fuzzy Math, 7: 997-1003, 1999.

[7] Y. L. Liu, S. Y. Liu and Y. Xu, ILI-ideals and prime $L I$-ideals in lattice implication algebras, Information Sciences, 105: 157-175, 2003.

[8] H. Zhu, Studing on the filters and ideals of lattice implication algebra, Southwest Jiaotong Univ, (in Chinese), 2005.

[9] Y. Xu and K. Y. Qin, On filters of lattice implication algebras, Fuzzy Math, 1: 251-260, 1993.

[10] K. Y. Qin and Y. Xu, The ultra filters of lattice implication algebras, Journal of South-west Jiaotong University, 34: 51-54, 1999.

[11] Y.B. Jun, Y. Xu and K.Y. Qin, Positive implicative and associative filters of lattice implication algebras, Bull. Korean Math. Soc. 35:53-61, 1998.

[12]J. Liu and Y. Xu, On the prime filters and decomposition theorem of lattice implication algebras, Fuzzy Math, 6: 1001-1008, 1998.

[13] Y.B. Jun, Implicative filters of lattice implication 
algebras, Bull. Korean Math. Soc. 34:193-198, 1997.

[14] J. Liu and Y. Xu, Filters and structure of lattice implication algebra, Chinese Sci. Bull. 42:1517-1520, 1997.

[15] Y. Xu, E.E. Kerre, D. Ruan and Z.M. Song, Fuzzy reasoning based on the extension principle, Int. J. Intelligent Syst. 16(4):469-495,2001.

[16] Y. Xu, K.Y. Qin, J. Liu and Z.M. Song, L-valued propositional logic $L_{v p l}$, Information Sci. 114:205-235, 1999.

[17] Y. Xu, D. Ruan, E.E. Kerre and J. Liu, $\partial$-Resolution principle based on first-order lattice-valued logic $L F(X)$, Information Sci. 132:221-239, 2001. 